\title{
||||||||||||||||||||||||||||||||||||||||||||||||||||||||||||||||||.
}

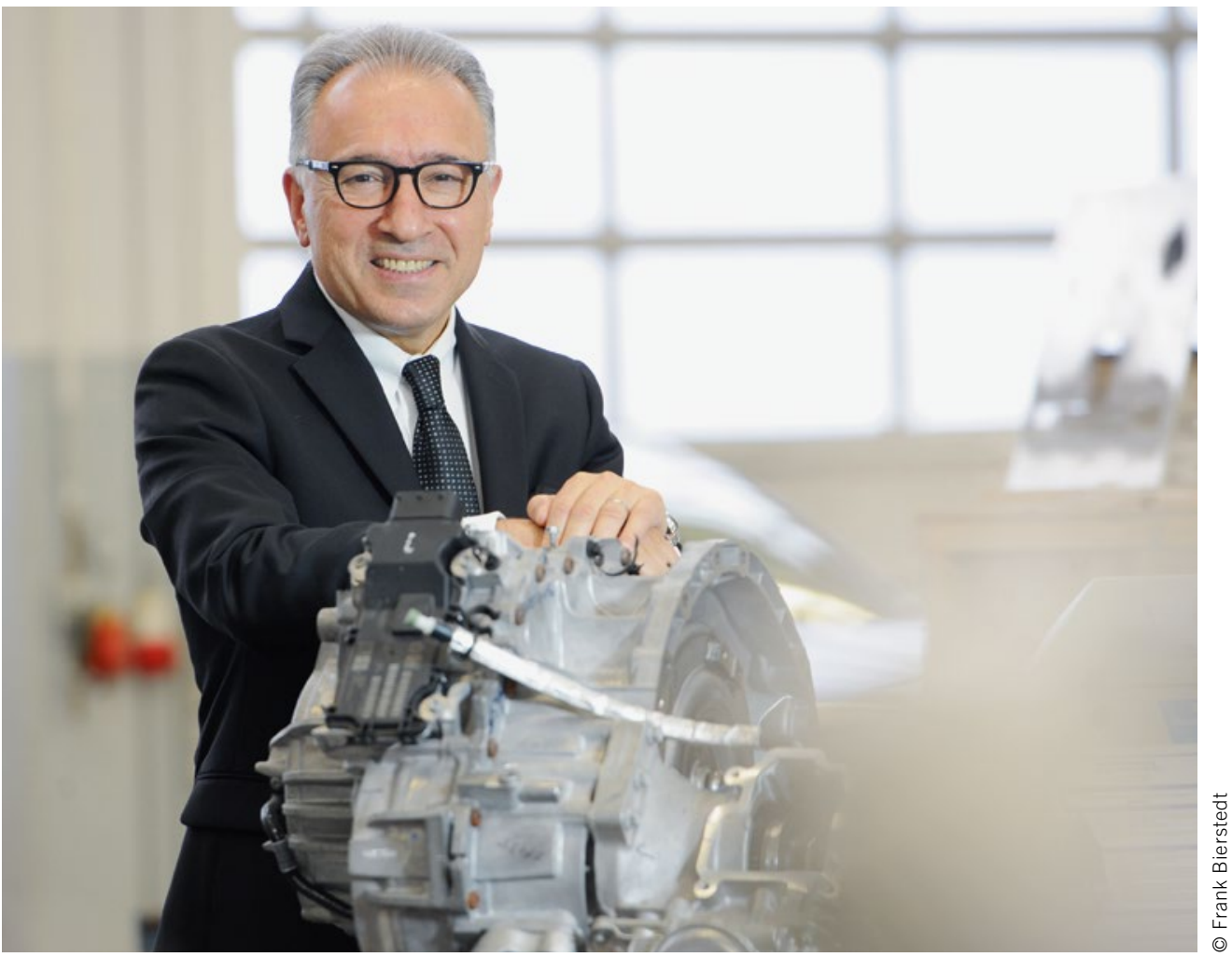

Prof. Dr.-Ing. Ferit Küçükay Director of the Institute of Automotive Engineering, Technical University of Braunschweig (Germany)

\section{Comparison of Hybrid Transmissions}

Dedicated Hybrid Transmissions (DHTs) are the most sold hybrid transmissions worldwide with a share of $70 \%$, followed by add-on hybrid transmissions - mostly in P2 configuration with 30 \%. Powersplit DHTs (PS-DHT), known from Toyota Prius, represent the majority of DHTs. The other part consists of multi-mode DHTs (MM-DHT), which for example have become known through the Voltec 2 from Chevrolet.

The word DHT was introduced as a new concept category for hybrid transmissions at the international CTI symposia on automotive transmissions, HEV and EV drives in Berlin in 2015 and in Detroit and Shanghai in 2016 and has become accepted among experts. A DHT describes a transmission that, in addition to the Internal Combustion Engine (ICE), only works in combination with one or more electric motors.

In case of add-on concepts for hybrid drives, an electric motor is added to an existing transmission concept to provide hybrid functions. This allows for hybrid drivetrains in P0 to P4 configurations. They can be scaled in terms of electric power, combined with $48 \mathrm{~V}$ or high voltage on-board power systems and are therefore feasible in different vehicle segments and different degrees of hybridisation. A disadvantage of a P2 hybrid is the comparatively poor mechanical efficiency due to the high number of gears and shift elements as well as actuators. Fur- thermore, the concept as parallel hybrid only allows for the variation of the ICE torque through the electric motor. The rotational speed is preset for each driving situation through the gear ratio.

In addition to the ICE torque, the ICE rotational speed can also be varied in PS-DHTs and MM-DHTs, which results in an optimisation of operating points and hence in improved drive efficiency; the mechanical efficiency of MM-DHTs is slightly lower than that of PS-DHTs due to the higher number of components with friction. However, if an ICE with identical power and driving performance requirements is used, a PS-DHT requires a higher electric motor power than MM-DHTs and P2 hybrids. Thus, it is possible with MM-DHTs - in addition to hybrid modes - to drive in purely electric or ICE modes with several gear ratios, which results in performance and efficiency advantages compared to PS-DHTs. Compared to P2 hybrids, however, PS-DHTs and MM-DHTs at present have a cost disadvantage. In future, it can be compensated by higher production volumes and decreasing costs of the electrical components.

In the course of the increasing electrification of drivetrains and in view of the expected - comparatively high - market shares of hybrid drives, the MM-DHTs in particular are an interesting and promising alternative. 